hep-th/yymmxxx

\title{
DOUBLE AFFINE HECKE ALGEBRA IN LOGARITHMIC CONFORMAL FIELD THEORY
}

\author{
G. MUTAFYAN AND I.YU. TIPUNIN
}

\begin{abstract}
We construct the representation of Double Affine Hecke Algebra whose symmetrization gives the center of the quantum group $\overline{\mathcal{U}}_{\mathfrak{q}} s \ell(2)$ and by Kazhdan-Lusztig duality the Verlinde algebra of $(1, p)$ models of logarithmic conformal field theory.
\end{abstract}

\section{INTRODUCTION}

Recently, quantum group methods led (see the recent review [1]) to a progress in logarithmic conformal field theory [2]. For the $(1, p)$ models [3], an equivalence between representation categories of the chiral algebra and the quantum group $\overline{\mathcal{U}}_{\mathfrak{q}} s \ell(2)$ was established [4] in the general framework of the Kazhdan-Lusztig duality [5]. Remarkably, the KL duality extends to an isomorphism between modular group representations on the quantum group center and on the space of generalized characters [6] of a $(1, p)$ model. Moreover, the Verlinde algebra of $(1, p)$ models [7, 8] (see also [9]) coincides [6] with the Grothendiec ring of $\bar{u}_{\mathfrak{q}} s \ell(2)$.

An unusual property of logarithmic conformal field theory is the nonsemisimplicity of the Verlinde algebra. However, this phenomenon does not look extraordinary in the Double affine Hecke algebra representation framework [11] of the Verlinde algebra classification. It leads to a natural conjecture [12] that the $(1, p)$ model Verlinde algebra can be realized in terms of a DAHA representation. Indeed, the representation of DAHA whose symmetrization gives the center of the quantum group $\bar{U}_{\mathfrak{q}} s \ell(2)$ and therefore the Verlinde algebra of $(1, p)$ models is identified in the present paper.

1.1. DAHA. We consider the symplest DAHA [11] generated by $X, Y$, and $T$ with the relations

$$
\begin{gathered}
T X T=X^{-1}, \quad T Y^{-1} T=Y, \quad X Y=\mathfrak{q} Y X T^{2}, \\
\left(T-\mathfrak{t}^{\frac{1}{2}}\right)\left(T+\mathfrak{t}^{-\frac{1}{2}}\right)=0, \quad \mathfrak{t}=\mathfrak{q}^{2} .
\end{gathered}
$$

In the paper we fix the deformation parameter

$$
\mathfrak{q}=e^{\frac{\mathbf{i} \pi}{p}}, \quad \mathfrak{q}^{\frac{1}{2}}=e^{\frac{\mathfrak{i} \pi}{2 p}},
$$

where $p=3,4,5,6, \ldots$ We let $\mathcal{H}$ denote this algebra. The group $P S L(2, \mathbb{Z})$ acts by automorphisms on $\mathcal{H}$

$$
\tau_{+}: \quad Y \rightarrow \mathfrak{q}^{-1 / 2} X Y, \quad X \rightarrow X, \quad T \rightarrow T
$$




$$
\tau_{-}: \quad X \rightarrow \mathfrak{q}^{1 / 2} Y X, \quad Y \rightarrow Y, \quad T \rightarrow T
$$

where

$$
\left(\begin{array}{ll}
1 & 1 \\
0 & 1
\end{array}\right) \rightarrow \tau_{+}, \quad\left(\begin{array}{ll}
1 & 0 \\
1 & 1
\end{array}\right) \rightarrow \tau_{-} .
$$

We note that the Fourier transform is given by

$$
\begin{aligned}
\sigma: \quad X & \rightarrow Y^{-1}, \quad Y \rightarrow X T^{2}, \quad T \rightarrow T, \\
\sigma & =\tau_{+} \tau_{-}^{-1} \tau_{+}=\tau_{-}^{-1} \tau_{+} \tau_{-}^{-1} .
\end{aligned}
$$

1.2. The representation. We consider a $6 p$-4-dimensional reducible but indecomposable representation $z$ of $\mathcal{H}$. The representation $z$ contains the maximal subrepresentation $V^{-2}$, which in notations of [11] is defined as the quotient $V^{-2}=\mathcal{P} /\left(X^{2 p}+X^{-2 p}-2\right)$, where $\mathcal{P}=\mathbb{C}\left[X, X^{-1}\right]$ is the standard representation of $\mathcal{H}$ in the Lourent polynomials. The $2 p$-4-dimensional irreducible quotient $\mathcal{M}=\mathcal{Z} / V^{-2}$ is isomorphic to the representation $V_{2 p-4}$ from [11] given by the quotient $\mathcal{P} / \varepsilon_{-p+2}$, where $\varepsilon_{-p+2}=\prod_{j=2}^{p-1}\left(\mathfrak{q}^{-j} X-\mathfrak{q}^{j} X^{-1}\right)$. We note also that $V^{-2}$ is also reducible and contains the maximal $2 p+4$-dimensional irreducible subrepresentation $\mathcal{W}$ and the quotient $\mathcal{E}=V^{-2} / \mathcal{W}$ is isomorphic to $\mathcal{M}$.

In Sec. 2, we describe the representation $Z$ by the explicit action of operators $T, X$ and $Y$ in a basis. Then we describe its structure and explicitly find the subrepresentation and quotients.

The representation $z$ bears a commutative associative multiplication, which is described in Sec. 1.5. The multiplication gives further the multiplication in the Verlinde algebra.

The $\operatorname{PSL}(2, \mathbb{Z})$ generators $\sigma$ and $\tau_{+}$are realized as a conjugation with some operators $\mathcal{S}$ and $\boldsymbol{v}$ respectively, acting in $\mathcal{Z}$. The operator $\boldsymbol{v}$ acts by a multiplication with an element from $Z$, which is abusing notation denoted by the same symbol $v$. By anology with [11] we call $v$ the Gaussian element.

1.3. Symmetrization. The operator $T$ has two different eigenvalues $\mathfrak{q}$ and $-\mathfrak{q}^{-1}$ in $z$. The eigenspace of $T$ with the eigenvalue $\mathfrak{q}$ is $3 p-1$-dimensional. We let $\mathcal{T}_{\mathfrak{q}}$ denote this eigenspace. In accordance with the general theory [11], $\mathcal{T}_{\mathfrak{q}}$ is an associative commutative algebra with multiplication induced by the multiplication in $Z$ and at the same time a representation of $S L(2, \mathbb{Z})$ induced by the $P S L(2, \mathbb{Z})$-action in $z$. The operators $\mathcal{S}, \boldsymbol{v}$, $C=-\left(X+X^{-1}\right)$ and $H=-\left(Y+Y^{-1}\right)$ have well defined restrictions on $\mathcal{T}_{\mathfrak{q}}$. Now we are ready to formulate the main result of the paper (the reader can find all needed quantum group definitions in [6]).

1.4. Theorem. - $\mathcal{T}_{\mathfrak{q}}$ is isomorphic to the center of $\overline{\mathcal{U}}_{\mathfrak{q}} s \ell(2)$ as associative commutative algebra and as $S L(2, \mathbb{Z})$ representation. 
- Under the isomorphism the eigenvectors of $C$ correspond to Radford images and eigenvectors of $H$ correspond to Drinfeld images of the characters of $\overline{\mathcal{U}}_{\mathfrak{q}} s \ell(2)$ irreducible reprsentations.

- The Gaussian element $\boldsymbol{v}$ coincides with the ribbon element of $\overline{\mathcal{U}}_{\mathfrak{q}} s \ell(2)$.

In Sec. 4, we describe the subspace of $T$ with the eigenvalue $\mathfrak{q}$, and in Sec. 5 we give the proof of theorem 1.4. The notations in this part correspond to the same notations in [6].

1.5. Structure of $z$. The very important information about the representation $z$ is encoded in the spectra of operators $X$ and $Y$. These operators are not diagonalizable but both have Jordan blocks of dimension 2. In order to describe their Jordan structure we introduce two basises in which operators $X$ and $Y^{-1}$ have a Jordan form. We call the first basis the $X$-basis and the second one the $Y$-basis. Jordan forms of $X$ and $Y^{-1}$ coincide in $Z$.

1.5.1. $X$-basis. The representation $Z$ has the basis

$$
w_{1} \ldots w_{2 p}, e_{1}, e_{p}, e_{p+1}, e_{2 p} ; \quad e_{2} \ldots e_{p-1}, e_{p+2} \ldots e_{2 p-1} ; \quad m_{2} \ldots m_{p-1}, m_{p+2} \ldots m_{2 p-1} \text {. }
$$

The subrepresentation $\mathcal{W}$ is spanned by elements $w_{1} \ldots w_{2 p}, e_{1}, e_{p}, e_{p+1}$ and $e_{2 p}$. The elements $e_{2} \ldots e_{p-1}, e_{p+2} \ldots e_{2 p-1}\left(m_{2} \ldots m_{p-1}, m_{p+2} \ldots m_{2 p-1}\right)$ give a basis in $\mathcal{E}$ (in $\mathcal{M})$ under the canonical projection. In basis (1.7) we have

$$
X w_{s}=\mathfrak{q}^{s} w_{s}, \quad X e_{s}=\mathfrak{q}^{s}\left(e_{s}+w_{s}\right), \quad X m_{s}=\mathfrak{q}^{s} m_{s}
$$

We call (1.7) the $X$-basis.

1.5.2. The multiplication in $z$. The representation $z$ is endowed with a commutative associative multiplication, which is naturally written in basis (1.7) as

$$
e_{i} e_{j}=\delta_{i, j} e_{j}, \quad e_{i} w_{j}=\delta_{i, j} w_{j}, \quad e_{i} m_{j}=\delta_{i, j} m_{j}, \quad w_{i} w_{j}=w_{i} m_{j}=m_{i} m_{j}=0
$$

1.5.3. $Y$-basis. The representation $z$ contains the basis

$$
u_{1} \ldots u_{2 p}, f_{1}, f_{p}, f_{p+1}, f_{2 p}, ; \quad f_{2} \ldots f_{p-1}, f_{p+2} \ldots f_{2 p-1} ; \quad k_{2} \ldots k_{p-1}, k_{p+2} \ldots k_{2 p-1}
$$

in which $Y^{-1}$ acts as follows

$$
Y^{-1} u_{s}=\mathfrak{q}^{s} u_{s}, \quad Y^{-1} f_{s}=\mathfrak{q}^{s}\left(f_{s}+u_{s}\right), \quad Y^{-1} k_{s}=\mathfrak{q}^{s} k_{s}
$$

We call (1.10) the $Y$-basis.

In Subsec. 2.3, we find the $Y$-basis and give decompositions of elements from the $Y$-basis in the $X$-basis. 
1.5.4. $P S L(2, \mathbb{Z})$-action. The operator $\mathcal{S}$ maps the $X$-basis to the $Y$-basis

$$
\mathcal{S} w_{s}=u_{s}, \quad \mathcal{S} e_{s}=f_{s}, \quad \mathcal{S} m_{s}=k_{s} .
$$

In Subsec. 3.1, we establish properties of the $\mathcal{S}$-operator. By a direct calculation, using the decompositions of the $Y$-basis in the $X$-basis, we check that this operator satisfies all relations (1.6) and $\mathcal{S}^{2}=\mathfrak{q} T^{-1}$.

In terms of the $X$-basis, the Gaussian element is

$$
\boldsymbol{v}=\sum_{s=1}^{2 p} \mathfrak{q}^{-\frac{1}{2}\left(s^{2}-1\right)} e_{s}-w_{1}+\mathfrak{q}^{-\frac{p^{2}}{2}} w_{p+1}+\left(\sum_{s=2}^{p-1}+\sum_{s=p+2}^{2 p-1}\right) \mathfrak{q}^{-\frac{1}{2}\left(s^{2}-1\right)}\left((p-s) w_{s}+p m_{s}\right) .
$$

The properties of this element are described in Subsec. 3.2.

In the end of Sec. 3, we prove the $P S L(2, \mathbb{Z})$ relations.

1.6. Notation. We introduce Chebyshov polynomials

$$
U_{s}(x)=x^{s-1}+x^{s-3}+\cdots+x^{-(s-3)}+x^{-(s-1)} .
$$

In what follows we often use the numbers

$$
[s, j] \equiv\left\{\begin{array} { l l } 
{ s , } & { j = 0 , 2 p , } \\
{ ( - 1 ) ^ { s - 1 } s , } & { j = p , } \\
{ \frac { [ s j ] } { [ j ] } , } & { j \operatorname { m o d } p \neq 0 , }
\end{array} \quad \{ s , j \} \equiv \left\{\begin{array}{ll}
0, & j \bmod p=0 \\
\frac{\{s j\}}{[j]} & \text { otherwise }
\end{array}\right.\right.
$$

\section{REPRESENTATION}

In this section, we recall the representation $V^{-2}$ [11] and then define the representation $Z$, which is an extension of $V^{-2}$. Then we find a Jordan basis for $Y$ in which $Y^{-1}$ acts by (1.11).

2.1. Polynomial representation $V^{-2}$. The representation $z$ is an extension of the representation $V^{-2}$ from [11]. To describe $V^{-2}$ we recall the standard representation [11] of $\mathcal{H}$ in the space of Laurent polynomials $\mathcal{P}=\mathbb{C}\left[X, X^{-1}\right]$. The $\mathcal{H}$ generators act as follows

$$
\begin{gathered}
T \rightarrow \mathfrak{t}^{\frac{1}{2}} \mathrm{~s}+\frac{\mathfrak{t}^{\frac{1}{2}}-\mathfrak{t}^{-\frac{1}{2}}}{X^{2}-1}(\mathrm{~s}-1), \quad \mathfrak{t}=\mathfrak{q}^{k}, \\
Y \rightarrow-\mathrm{sp} T
\end{gathered}
$$


where

$$
\mathrm{s} f(X)=f\left(X^{-1}\right), \quad \text { p } f(X)=f(\mathfrak{q} X)
$$

and $X, X^{-1}$ act by multiplication. (We note that these formulas differ from [11] by the sign in the definition of $Y \rightarrow \operatorname{sp} T$.) The representation $V^{-2}$ is the $4 p$-dimensional representation in the quotient space $\mathcal{P} /\left(X^{2 p}+X^{-2 p}-2\right)$.

2.1.1. Proposition. - The operators $X$ and $Y$ have in $V^{-2}$ eigenvalues $\mathfrak{q}^{s}, s=$ $1 \ldots 2 p$, each with multiplicity 2.

- The Jordan basis of $X$ contains functions $e_{s}$ and $w_{s}$ for $s=1 \ldots 2 p$.

- The Jordan basis of $Y$ contains functions $u_{s}$ for $s=1 \ldots 2 p$ and $k_{s}$ for $s=$ $2 \ldots p-1, p+2 \ldots 2 p-1$, and functions $f_{1}, f_{p}, f_{p+1}, f_{2 p}$.

The action of $X$ and $Y^{-1}$ in these basises is

$$
\begin{gathered}
X w_{s}=\mathfrak{q}^{s} w_{s}, \quad X e_{s}=\mathfrak{q}^{s}\left(e_{s}+w_{s}\right), \\
Y^{-1} u_{s}=\mathfrak{q}^{s} u_{s}, \quad Y^{-1} f_{s}=\mathfrak{q}^{s}\left(f_{s}+u_{s}\right), \quad Y^{-1} k_{s}=\mathfrak{q}^{s} k_{s} .
\end{gathered}
$$

Proof. To describe the spectra of these operators, we introduce functions

$$
\begin{aligned}
& w_{s}=\frac{1}{4 p^{2}}\left(X^{2 p}-1\right) \sum_{j=0}^{2 p-1} \mathfrak{q}^{-s j} X^{j}, \quad s=1 \ldots 2 p, \\
& e_{s}=\frac{1}{2 p}+\frac{1}{4 p^{2}} \sum_{j=1}^{2 p-1}(2 p-j)\left(\mathfrak{q}^{-s j} X^{j}+\mathfrak{q}^{s j} X^{-j}\right),
\end{aligned}
$$

and

$$
\begin{aligned}
& u_{s}=\frac{(-1)^{s}}{p \sqrt{2 p}}\left(\mathfrak{q}^{s} \frac{U_{p-s}(X)+U_{p+s}(X)}{2}+\mathfrak{q} \frac{U_{p-s}\left(\mathfrak{q}^{-1} X\right)+U_{p+s}\left(\mathfrak{q}^{-1} X\right)}{2}\right), \\
& u_{p+s}=\frac{(-1)^{p+s}}{p \sqrt{2 p}}\left(\mathfrak{q}^{p+s} \frac{U_{s}(X)+U_{2 p-s}(X)}{2}+\mathfrak{q} \frac{U_{s}\left(\mathfrak{q}^{-1} X\right)+U_{2 p-s}\left(\mathfrak{q}^{-1} X\right)}{2}\right) \text {, } \\
& k_{s}=\frac{(-1)^{s+1}}{p \sqrt{2 p}}\left(\mathfrak{q}^{s} U_{p-s}(X)+\mathfrak{q} U_{p-s}\left(q^{-1} X\right)\right), \\
& k_{p+s}=\frac{(-1)^{p+s}}{p \sqrt{2 p}}\left(\mathfrak{q}^{p+s} U_{s}(X)+\mathfrak{q} U_{s}\left(q^{-1} X\right)\right) \text {, } \\
& s=2 \ldots p-1 \\
& f_{s}=\frac{1}{p \sqrt{2 p}} \cdot\left\{\begin{array}{lr}
\frac{(-1)^{p+1} U_{2 p}(X)}{2}, & s=p, \\
U_{p}(X), & s=2 p, \\
\frac{(-1)^{p} \mathfrak{q} X U_{2 p}(X)}{2}, & s=p+1, \\
-q X U_{p}(X), & s=1 .
\end{array}\right. \\
& s=1 \ldots p,
\end{aligned}
$$


Then, (2.4) is checked by a direct calculation. It is easy to see that $1=\sum_{s=1}^{2 p} e_{s}$. Together with (2.4) this gives

$$
X^{j}=\sum_{s=1}^{2 p} \mathfrak{q}^{s j}\left(e_{s}+j w_{s}\right), j=0, \pm 1, \pm 2 \ldots
$$

i.e. functions $e_{s}$ and $w_{s}$ are linearly independent and form a basis in $V^{-2}$.

(2.5) is also checked by a direct calculation using the following relations

$$
\begin{gathered}
Y U_{s}\left(q^{-1} X\right)=\left(\mathfrak{q}^{s}+\mathfrak{q}^{-s}\right) U_{s}\left(q^{-1} X\right)-q^{-1} U_{s}(X), \\
Y U_{s}(X)=\mathfrak{q} U_{s}\left(q^{-1} X\right) .
\end{gathered}
$$

The linear independence of these vectors is proved by the standard technic (See definition 2.5.4 and theorems 2.5.9 and 2.9.3 from [11].).

The representation $V^{-2}$ is reducible. It has a $2 p+4$-dimensional subrepresenation $\mathcal{W}$ spanned by functions $w_{1} \ldots w_{2 p}, e_{1}, e_{p}, e_{p+1}$ and $e_{2 p}$. The quotient $\mathcal{E}=V^{-2} / \mathcal{W}$ is isomorphic to $V_{2 p-4}$ from [11]. The representation $V_{2 p-4}$ is defined in [11] as the quotient $V_{2 p-4}=\mathcal{P} / \varepsilon_{-p+2}$, where $\varepsilon_{-p+2}=\prod_{j=2}^{p-1}\left(\mathfrak{q}^{-j} X-\mathfrak{q}^{j} X^{-1}\right)$.

Decomposition of any polynomial $f(X)$ in the basis $e_{s}, w_{s}$ is given by

$$
f(X)=\sum_{s=1}^{2 p}\left(f\left(\mathfrak{q}^{s}\right) e_{s}+\left(\left.X \frac{d f(X)}{d X}\right|_{X=\mathfrak{q}^{s}}\right) w_{s}\right) .
$$

Using it, we check that $u_{1} \ldots u_{2 p}, f_{1}, f_{p}, f_{p+1}$ and $f_{2 p}$ belong to $\mathcal{W}$ and therefore in $\mathcal{W}$ Jordan forms of $X$ and $Y^{-1}$ coincide. But in the whole $V^{-2}$ they do not coincide, hence automorphism (1.6) cannot be realized as a conjugation. To recover this, we find an extension of $V^{-2}$ to a $6 p-4$-dimensional representation $z$ by adding vectors $m_{2}, \ldots, m_{p-1}$ and $m_{p+2}, \ldots, m_{2 p-1}$. The action of $X$ on them is $X m_{s}=\mathfrak{q}^{s} m_{s}$. The whole $z$ cannot be realized in a space of polynomials in 1 varible. We describe $Z$ in terms of an abstract vector space.

2.2. The representation $z$ in the $X$-basis. We assume the following definition of $z$. The representation $Z$ is a $6 p-4$-dimensional vector space with the basis consisting of $4 p$ vectors $e_{s}$ and $w_{s}$ with $s=1 \ldots 2 p$, and $2 p-4$ vectors $m_{s}$ with $s=2 \ldots p-1, p+$ $2 \ldots 2 p-1$. The action of $\mathcal{H}$-operators in this basis is defined by the formulas:

$$
\begin{gathered}
X w_{s}=\mathfrak{q}^{s} w_{s}, \quad X e_{s}=\mathfrak{q}^{s}\left(e_{s}+w_{s}\right), \quad X m_{s}=\mathfrak{q}^{s} m_{s}, \\
T w_{p}=-q^{-1} w_{p}-\left(q-q^{-1}\right) e_{p}, \quad T w_{2 p}=-q^{-1} w_{2 p}-\left(q-q^{-1}\right) e_{2 p}, \\
T w_{s}=-\frac{\mathfrak{q}^{-s}}{[s]} w_{s}-\frac{[s-1]}{[s]} w_{2 p-s}, \quad s \neq 0, p, \\
T e_{p}=\mathfrak{q} e_{p}, \quad T e_{2 p}=\mathfrak{q} e_{2 p},
\end{gathered}
$$




$$
Y e_{s}=\frac{\mathfrak{q}^{-s}}{[s]} e_{2 p-s+1}-\frac{[s-1]}{[s]} e_{s+1}-\frac{2}{\left(q-q^{-1}\right)[s]^{2}}\left(w_{s+1}-w_{2 p-s+1}\right), \quad s \neq 0, p,
$$

$$
\begin{gathered}
Y m_{2 p-1}=-\mathfrak{q} m_{2}-\left(\mathfrak{q}+\mathfrak{q}^{-1}\right) w_{0}, \quad Y m_{p-1}=-\mathfrak{q} m_{p+2}, \\
Y m_{s}=-\frac{[s-1]}{[s]} m_{s+1}+\frac{\mathfrak{q}^{-s}}{[s]} m_{2 p-s+1}, \quad s=2 \ldots p-2, p+2 \ldots 2 p-2 .
\end{gathered}
$$

We note that the basis $e, w, m$ by definition is the $X$-basis (1.7) and (2.11) gives the Jordan structure of $X$.

2.2.1. Lemma. Operators $X, Y$ and $T$ defined by (2.11)-(2.24) satisfy the DAHA relations (1.1) and (1.2).

Proof. A direct calculation.

We define a commutative associative multiplication in $z$ by formulas (1.9).

2.2.2. Proposition. $Z$ is reducible. The $2 p+4$-dimensional subspace

$$
\mathcal{W} \equiv\left\{w_{1} \ldots w_{2 p}, e_{1}, e_{p}, e_{p+1}, e_{2 p}\right\}
$$

is invariant under the $\mathcal{H}$-action and is therefore a subrepresentation. The quotient is a direct sum: $\mathcal{Z} / \mathcal{W}=\mathcal{E} \oplus \mathcal{M}$, where $\mathcal{E} \equiv\left\{e_{2} \ldots e_{p-1}, e_{p+2} \ldots e_{2 p-1}\right\}$ and $\mathcal{M} \equiv\left\{m_{2} \ldots m_{p-1}\right.$, $\left.m_{p+2} \ldots m_{2 p-1}\right\}$.

Proof. Immediately follows from (2.11)-2.24).

2.3. $Y$-basis. In this subsection we prove that the Jordan form of $Y$ is (1.11).

2.3.1. Proposition. A Jordan basis of $Y$ consists of $6 p-4$ vectors: $4 p$ vectors $f_{s}, u_{s}$ for $s=1, \ldots, 2 p$, and $2 p-4$ vectors $k_{s}$ for $s=2, \ldots, p-1, p+2 \ldots 2 p-1$. The action of $Y^{-1}$ on this vectors is given by (1.11). 
Proof. We define in the $X$-basis the vectors

$$
u_{s}=\sum_{j=1}^{2 p} \mathrm{u}_{j, s}^{(w)} w_{j}+\sum_{j=1}^{2 p} \mathrm{u}_{j, s}^{(e)} e_{j}, \quad s=1 \ldots 2 p,
$$

where coefficients are

$$
\begin{aligned}
\mathrm{u}_{j, s}^{(w)} & =\frac{(-1)^{s+j}}{\sqrt{2 p}}\left(\mathfrak{q}^{s}\{s, j\}-\mathfrak{q}\{s, j-1\}\right), \quad j=1 \ldots 2 p ; \\
\mathrm{u}_{1, s}^{(e)} & =(-1)^{s} \frac{\mathfrak{q}}{\sqrt{2 p}} ; \quad \mathrm{u}_{2 p, s}^{(e)}=(-1)^{s} \frac{\mathfrak{q}^{s}}{\sqrt{2 p}} ; \\
\mathrm{u}_{p+1, s}^{(e)} & =(-1)^{p+1} \frac{\mathfrak{q}}{\sqrt{2 p}} ; \quad \mathrm{u}_{p, s}^{(e)}=(-1)^{p+1} \frac{\mathfrak{q}^{s}}{\sqrt{2 p}} ; \\
\mathrm{u}_{j, s}^{(e)} & =0, \quad j \neq 1, p, p+1,2 p,
\end{aligned}
$$

the vectors

$$
k_{s}=\sum_{j=1}^{2 p} \mathrm{k}_{j, s}^{(w)} w_{j}+\sum_{j=1}^{2 p} \mathrm{k}_{j, s}^{(e)} e_{j}, \quad s=2 \ldots p-1, p+2 \ldots 2 p-1,
$$

where coefficients are

$$
\begin{aligned}
\mathrm{k}_{j, s}^{(w)} & =-\frac{(p-s)}{p} \mathrm{u}_{j, s}^{(w)}-\frac{(-1)^{s+j}}{p \sqrt{2 p}}\left(\mathfrak{q}^{s}[s, j]\{1, j\}-\mathfrak{q}[s, j-1]\{1, j-1\}\right), \\
\mathrm{k}_{1, s}^{(e)} & =(-1)^{s+1} \frac{\left(q^{s}[s]+q(p-s)\right)}{p \sqrt{2 p}}, \quad \mathrm{k}_{p, s}^{(e)}=(-1)^{p} \frac{\left(q[s]+q^{s}(p-s)\right)}{p \sqrt{2 p}}, \\
\mathrm{k}_{p+1, s}^{(e)} & =(-1)^{p} \frac{\left(q^{s}[s]+q(p-s)\right)}{p \sqrt{2 p}}, \quad \mathrm{k}_{2 p, s}^{(e)}=(-1)^{s+1} \frac{\left(q[s]+q^{s}(p-s)\right)}{p \sqrt{2 p}}, \\
\mathrm{k}_{j, s}^{(e)} & =\frac{(-1)^{s+j}}{p \sqrt{2 p}}\left(\mathfrak{q}^{s}[s, j]-\mathfrak{q}[s, j-1]\right) \quad j \neq 1, p, p+1,2 p,
\end{aligned}
$$

and the vectors

$$
f_{s}=\sum_{j=1}^{2 p} \mathrm{f}_{j, s}^{(w)} w_{j}+\sum_{j=1}^{2 p} \mathrm{f}_{j, s}^{(e)} e_{j}+\left(\sum_{j=2}^{p-1}+\sum_{j=p+2}^{2 p-1}\right) \mathrm{f}_{j, s}^{(m)} m_{j}, \quad s=1 \ldots 2 p,
$$

where coefficients are

$$
\begin{aligned}
\mathrm{f}_{1, s}^{(w)} & =\frac{2(-1)^{s+1} \mathfrak{q}^{2 s}}{\left(\mathfrak{q}-\mathfrak{q}^{-1}\right) \sqrt{2 p}}, \quad \mathrm{f}_{p, s}^{(w)}=\frac{\mathfrak{q}(-1)^{p+1}[s]}{\sqrt{2 p}}, \quad \mathrm{f}_{p+1, s}^{(w)}=\frac{2(-1)^{p} \mathfrak{q}^{2 s}}{\left(\mathfrak{q}-\mathfrak{q}^{-1}\right) \sqrt{2 p}}, \\
\mathrm{f}_{2 p, s}^{(w)} & =\frac{\mathfrak{q}(-1)^{s}[s]}{\sqrt{2 p}}, \\
\mathrm{f}_{j, s}^{(w)} & =-p(p-j) \mathrm{k}_{j, s}^{(e)}+\frac{(-1)^{s+j}}{\sqrt{2 p}}\left(\mathfrak{q}[s, j-1]+\mathfrak{q}^{s}\{s, j\}\right), \quad j \neq 1, p, p+1,2 p, \\
\mathrm{f}_{p, s}^{(e)} & =(-1)^{p+s+1} \mathrm{f}_{2 p, s}^{(e)}=(-1)^{p+1} \frac{\mathfrak{q}^{s}}{\sqrt{2 p}}, \quad \mathrm{f}_{j, s}^{(e)}=0 \quad j \neq p, 2 p, \\
\mathrm{f}_{j, s}^{(m)} & =-p^{2} \mathrm{k}_{j, s}^{(e)}, \quad j \neq 1, p, p+1,2 p
\end{aligned}
$$


and the coefficient $\mathrm{f}_{j, s}^{(m)}$ in (2.30) is 0 for $s=1, p, p+1,2 p$.

Then, (1.11) is checked by a simple calculation using formulas (2.19)-(2.24).

The linear independence of these vectors is proved in the following way. From the decompositions in the $X$-basis, we obtain that vectors $u_{s}, f_{1}, f_{p}, f_{p+1}, f_{2 p}$ belong to $\mathcal{W}$, vectors $k_{s}$ belong to $\mathcal{W}+\mathcal{E}$, and vectors $f_{s}$ with $s=2 \ldots p-1, p+2 \ldots 2 p-1$ belong to $\mathcal{W}+$ $\mathcal{M}$. We recall, that under the isomotphism $\mathcal{W}+\mathcal{E} \sim V^{-2}$ vectors $u_{s}, k_{s}, f_{1}, f_{p}, f_{p+1}, f_{2 p}$ correspond to the linearly independent functions (2.7)-(2.9) in $V^{-2}$, and therefore the vectors are also linearly independent. In particular, vectors $u_{s}, f_{1}, f_{p}, f_{p+1}, f_{2 p}$ form a basis in $\mathcal{W}$ and therefore images of the vectors $k_{s}$ under the canonical projection to $\mathcal{E}=(\mathcal{W}+\mathcal{E}) / \mathcal{W}$ form a basis in $\mathcal{E}$. We let abusing notations $k_{s}$ denote these images. We recall that the isomorphism $\mathcal{E} \sim \mathcal{M}$ maps vectors $k_{s}$ (images under the canonical projections of $k_{s} \in Z$ ) to $f_{s}$ (images under the canonical projections of $f_{s} \in Z$ ), and therefore $f_{s}$ with $s=2 \ldots p-1, p+2 \ldots 2 p-1$ are linearly independent. Thus, the linear independence of all vectors $u, f, k$ is established.

\section{3. $\operatorname{PSL}(2, \mathbb{Z})$ ACTION IN $Z$}

In this section we define operators $\mathcal{S}$ and $\boldsymbol{v}$ and prove that they satisfy $P S L(2, \mathbb{Z})$ relations. Conjugations with operators $\mathcal{S}$ and $\boldsymbol{v}$ give automorphisms $\sigma$ and $\tau_{+}$respectively.

3.1. $\sigma$. We define the $\mathcal{S}$-operator that maps the $X$-basis to the $Y$-basis by formulas $(1.12)$.

3.1.1. Proposition. $\mathcal{S}$ satisfies relations

$$
\begin{gathered}
\mathcal{S} X \mathcal{S}^{-1}=Y^{-1}, \\
\mathcal{S} Y \mathcal{S}^{-1}=X T^{2}, \\
\mathcal{S} T \mathcal{S}^{-1}=T, \\
\mathcal{S}^{2}=\mathfrak{q} T^{-1} .
\end{gathered}
$$

Proof. $\quad-13.1$ follows from the definition of $\mathcal{S}$.

- (3.4) follows from a direct calculation of $T \mathcal{S}^{2}$-action in the $X$-basis. We give a detailed calculation of $T \mathcal{S}^{2} e_{s}$. The calculation of $T \mathcal{S}^{2} w_{s}$ and $T \mathcal{S}^{2} m_{s}$ is similar and is omitted. We check that $T \mathcal{S}^{2} e_{s}=\mathfrak{q} e_{s}$. We begin with

$$
\begin{gathered}
\mathcal{S}^{2} e_{s}=\mathcal{S} f_{s}=\mathcal{S}\left(\sum_{r=1}^{2 p} \mathrm{f}_{r, s}^{(w)} w_{r}+\mathrm{f}_{p, s}^{(e)} e_{p}+\mathrm{f}_{2 p, s}^{(e)} e_{2 p}+\left(\sum_{r=2}^{p-1}+\sum_{r=p+2}^{2 p-1}\right) \mathrm{f}_{r, s}^{(m)} m_{r}\right)= \\
=\mathrm{f}_{1, s}^{(w)} u_{1}+\mathrm{f}_{p, s}^{(w)} u_{p}+\mathrm{f}_{p+1, s}^{(w)} u_{p+1}+\mathrm{f}_{2 p, s}^{(w)} u_{2 p}+\mathrm{f}_{p, s}^{(e)} f_{p}+\mathrm{f}_{2 p, s}^{(e)} f_{2 p}+ \\
+\left(\sum_{r=2}^{p-1}+\sum_{r=p+2}^{2 p-1}\right)\left(\mathrm{f}_{r, s}^{(w)} u_{r}+\mathrm{f}_{r, s}^{(m)} k_{r}\right) .
\end{gathered}
$$


Then we calculate coefficients in front of $e_{j}$ and $w_{j}$ in (3.5) using (2.26)-(2.31). This calculation is cumbersome and is given in Appendix A. The result of the calculation is

$$
\begin{gathered}
\mathcal{S}^{2} e_{s}=-\frac{\mathfrak{q}^{s+1}}{[s]} e_{s}+\mathfrak{q} \frac{[s-1]}{[s]} e_{2 p-s}+\frac{2\left(\mathfrak{q}^{2}-1\right)}{\left(\mathfrak{q}^{s}-\mathfrak{q}^{-s}\right)^{2}}\left(w_{s}-w_{2 p-s}\right), \quad s \neq p, 2 p, \\
\mathcal{S}^{2} e_{p}=e_{p}, \quad \mathcal{S}^{2} e_{2 p}=e_{2 p} .
\end{gathered}
$$

A simple calculation using (2.12) $-(2.15)$ gives

$$
T \mathcal{S}^{2} e_{s}=\mathfrak{q} e_{s}, \quad s=1 \ldots 2 p .
$$

- (3.3) is checked as follows $\mathcal{S}^{2}=\mathfrak{q} T^{-1} \Rightarrow \mathcal{S} T=T \mathcal{S}\left(=\mathfrak{q} \mathcal{S}^{-1}\right) \Rightarrow S S^{-1}=T$.

- (3.2) is checked as follows $\mathcal{S} X \mathcal{S}^{-1} \stackrel{\text { 3.1. }}{=} Y^{-1} \Rightarrow \mathcal{S} X^{-1} \mathcal{S}^{-1}=Y \Rightarrow \mathcal{S} Y \mathcal{S}^{-1}=$ $\mathcal{S}^{2} X^{-1} \mathcal{S}^{-2} \stackrel{\text { 3.4. }}{=} T^{-1} X^{-1} T \stackrel{\text { 1.1. }}{=} X T^{2}$.

3.2. $\tau_{+}$. The automorphism $\tau_{+}$can be realized as a conjugation with the element $\boldsymbol{v} \in Z$ given by (1.13).

3.2.1. Proposition. For $\boldsymbol{v}$ given by (1.13), the operator

$$
\tau_{+}(x)=\boldsymbol{v}^{-1} x \boldsymbol{v}, \quad \forall x \in \mathcal{H}
$$

satisfies relations (1.4).

Proof. A direct calculation.

3.2.2. Proposition. The map

$$
\left(\begin{array}{cc}
0 & 1 \\
-1 & 0
\end{array}\right) \rightarrow \mathcal{S}, \quad\left(\begin{array}{ll}
1 & 1 \\
0 & 1
\end{array}\right) \rightarrow \boldsymbol{v}
$$

gives a $P S L(2, \mathbb{Z})$ action in $z$.

Proof. Relations (3.1)-(3.4) and (1.4) are sufficient [11] to check the $P S L(2, \mathbb{Z})$ relations.

\section{EIGENSPACE OF $T$ WITH EIGENVALUE $\mathfrak{q}$}

In this section, we describe the representation of the symmetrized DAHA. In section 5, we prove that it is isomorphic to the centre of $\overline{\mathcal{U}}_{\mathfrak{q}} s \ell(2)$.

We let $\mathcal{T}_{\mathfrak{q}}$ denote the eigenspace of $T$ with the eigenvalue $\mathfrak{q}$. It is $3 p-1$ dimensional. Operators $X$ and $Y$ have no well-defined restriction to $\mathcal{T}_{\mathfrak{q}}$ but "symmetrized" operators $C=-\left(X+X^{-1}\right)$ and $H=-\left(Y+Y^{-1}\right)$ have. Indeed, for a given $\mathbf{a} \in \mathcal{T}_{\mathfrak{q}}$, we have

$$
T\left(X+X^{-1}\right) \mathbf{a} \stackrel{[1.1}{=}\left(X^{-1} T^{-1}+T X^{-1}\right) \mathbf{a}=\left(\mathfrak{q}^{-1}+T\right) X^{-1} \mathbf{a} \stackrel{[1.2]}{=}
$$




$$
=\left(\mathfrak{q}+T^{-1}\right) X^{-1} \mathbf{a}=\left(\mathfrak{q} X^{-1}+X T\right) \mathbf{a}=\mathfrak{q}\left(X+X^{-1}\right) \mathbf{a} .
$$

Thus, $\left(X+X^{-1}\right) \mathbf{a} \in \mathcal{T}_{\mathfrak{q}}$ and a similar calculation shows that $H$ has the well-defined restriction to $\mathcal{T}_{\mathfrak{q}}$ as well.

4.1. $C$-basis. The eigenvectors of $C=-\left(X+X^{-1}\right)$ are

$$
\begin{gathered}
\boldsymbol{e}_{0}=e_{p}, \quad \boldsymbol{e}_{p}=e_{2 p}, \quad \boldsymbol{e}_{s}=e_{p+s}+e_{p-s}, \\
\boldsymbol{w}_{1}^{+}=\frac{1}{\mathfrak{q}-\mathfrak{q}^{-1}} m_{p-1}, \quad \boldsymbol{w}_{1}^{-}=\frac{1}{\mathfrak{q}-\mathfrak{q}^{-1}}\left(w_{p+1}-w_{p-1}-m_{p-1}\right), \\
\boldsymbol{w}_{s}^{+}=\frac{[s]}{\mathfrak{q}-\mathfrak{q}^{-1}}\left(m_{p-s}+m_{p+s}\right), \quad \boldsymbol{w}_{s}^{-}=\frac{[s]}{\mathfrak{q}-\mathfrak{q}^{-1}}\left(w_{p+s}-w_{p-s}-m_{p-s}-m_{p+s}\right), \\
\boldsymbol{w}_{p-1}^{+}=\frac{1}{\mathfrak{q}-\mathfrak{q}^{-1}}\left(m_{2 p-1}-w_{1}\right), \quad \boldsymbol{w}_{p-1}^{-}=\frac{1}{\mathfrak{q}-\mathfrak{q}^{-1}}\left(w_{2 p-1}-m_{2 p-1}\right), \\
\boldsymbol{w}_{s}=\boldsymbol{w}_{s}^{+}+\boldsymbol{w}_{s}^{-}=\frac{[s]}{\mathfrak{q}-\mathfrak{q}^{-1}}\left(w_{p+s}-w_{p-s}\right), \quad s=1 \ldots p-1 .
\end{gathered}
$$

The action of $C$ on them folows from (2.11)

$$
\begin{gathered}
C \boldsymbol{e}_{0}=\mu_{0} \boldsymbol{e}_{0}, \quad C \boldsymbol{e}_{p}=\mu_{p} \boldsymbol{e}_{p}, \\
C \boldsymbol{e}_{s}=\mu_{s} \boldsymbol{e}_{s}+\left(\mathfrak{q}-\mathfrak{q}^{-1}\right)^{2} \boldsymbol{w}_{s}, \quad s=1 \ldots p-1 \\
C \boldsymbol{w}_{s}^{ \pm}=\mu_{s} \boldsymbol{w}_{s}^{ \pm}, \quad s=1 \ldots p-1,
\end{gathered}
$$

where

$$
\mu_{s}=\mathfrak{q}^{s}+\mathfrak{q}^{-s}, \quad 0 \leqslant s \leqslant p .
$$

The multiplication in $\mathcal{T}_{\mathfrak{q}}$ is induced by (1.9)

$$
\boldsymbol{e}_{r} \boldsymbol{w}_{s}^{ \pm}=\delta_{r, s} \boldsymbol{w}_{s}^{ \pm}, \quad \boldsymbol{e}_{r} \boldsymbol{e}_{s}=\delta_{r, s} \boldsymbol{e}_{s}, \quad \boldsymbol{w}_{r}^{ \pm} \boldsymbol{w}_{s}^{ \pm}=0
$$

4.2. $H$-basis. The eigenvectors of $H=-\left(Y+Y^{-1}\right)$ are

$$
\begin{gathered}
\boldsymbol{f}_{0}=f_{p}, \quad \boldsymbol{f}_{p}=f_{0}, \quad \boldsymbol{f}_{s}=f_{p+s}+f_{p-s}, \\
\boldsymbol{u}_{1}^{+}=\frac{1}{\mathfrak{q}-\mathfrak{q}^{-1}} k_{p-1}, \quad \boldsymbol{u}_{1}^{-}=\frac{1}{\mathfrak{q}-\mathfrak{q}^{-1}}\left(u_{p+1}-u_{p-1}-k_{p-1}\right), \\
\boldsymbol{u}_{s}^{+}=\frac{[s]}{\mathfrak{q}-\mathfrak{q}^{-1}}\left(k_{p-s}+k_{p+s}\right), \quad \boldsymbol{u}_{s}^{-}=\frac{[s]}{\mathfrak{q}-\mathfrak{q}^{-1}}\left(u_{p+s}-u_{p-s}-k_{p-s}-k_{p+s}\right), \\
\boldsymbol{u}_{p-1}^{+}=\frac{1}{\mathfrak{q}-\mathfrak{q}^{-1}}\left(k_{2 p-1}-u_{1}\right), \quad \boldsymbol{u}_{p-1}^{-}=\frac{1}{\mathfrak{q}-\mathfrak{q}^{-1}}\left(u_{2 p-1}-k_{2 p-1}\right), \\
\boldsymbol{u}_{s}=\boldsymbol{u}_{s}^{+}+\boldsymbol{u}_{s}^{-}=\frac{[s]}{\mathfrak{q}-\mathfrak{q}^{-1}}\left(u_{p+s}-u_{p-s}\right), \quad s=1 \ldots p-1 .
\end{gathered}
$$

The action of $H$ on them folows from (1.11)

$$
\begin{gathered}
H \boldsymbol{f}_{0}=\mu_{0} \boldsymbol{f}_{0}, \quad H \boldsymbol{f}_{p}=\mu_{p} \boldsymbol{f}_{p} \\
H \boldsymbol{f}_{s}=\mu_{s} \boldsymbol{f}_{s}+\left(\mathfrak{q}-\mathfrak{q}^{-1}\right)^{2} \boldsymbol{u}_{s}, \quad s=1 \ldots p-1,
\end{gathered}
$$




$$
H \boldsymbol{u}_{s}^{ \pm}=\mu_{s} \boldsymbol{u}_{s}^{ \pm}, \quad s=1 \ldots p-1,
$$

where eigenvalues are given by (4.5).

4.3. $S L(2, \mathbb{Z})$ action. Operators $\mathcal{S}$ and $\boldsymbol{v}$ have well-defined restrictions to $\mathcal{T}_{\mathfrak{q}}$. This endows $\mathcal{T}_{\mathfrak{q}}$ with a representation of $S L(2, \mathbb{Z})$. In more detail, $\mathcal{S}$-operator in $\mathcal{T}_{\mathfrak{q}}$ satisfies

$$
\begin{aligned}
\mathcal{S} \boldsymbol{e}_{s} & =\boldsymbol{f}_{s}, \quad s=0 \ldots p, \\
\mathcal{S} \boldsymbol{w}_{s}^{ \pm} & =\boldsymbol{u}_{s}^{ \pm}, \quad s=1 \ldots p-1
\end{aligned}
$$

and because $T=\mathfrak{q}$ in $\mathcal{T}_{\mathfrak{q}}$, we have $\mathcal{S}^{2}=1$. We note also that in $\mathcal{T}_{\mathfrak{q}}$ relations 3.1 and 3.2 lead to

$$
\mathcal{S} C \mathcal{S}^{-1}=H
$$

\section{Proof of Theorem 1.4}

We note that $\mathcal{T}_{\mathfrak{q}}$ and the center of $\overline{\mathcal{U}}_{\mathfrak{q}} s \ell(2)$ from [6] have the same dimension equal to $3 p-1$. Then we identify $C$-basis ( $H$-basis) in $\mathcal{T}_{\mathfrak{q}}$ with the Radford images (Drinfeld images) of $q$-characters of irreducible representations

$$
\begin{aligned}
& \widehat{\boldsymbol{\phi}}^{+}(s)=\omega_{s} \boldsymbol{w}_{s}^{+}, \quad \widehat{\boldsymbol{\phi}}^{-}(s)=\omega_{p-s} \boldsymbol{w}_{p-s}^{-}, \quad s=1 \ldots p-1, \\
& \widehat{\boldsymbol{\phi}}^{+}(p)=p \sqrt{2 p} \boldsymbol{e}_{p}, \quad \widehat{\boldsymbol{\phi}}^{-}(p)=(-1)^{p+1} p \sqrt{2 p} \boldsymbol{e}_{0}, \\
& \boldsymbol{\chi}^{+}(s)=\omega_{s} \boldsymbol{u}_{s}^{+}, \quad \boldsymbol{\chi}^{-}(s)=\omega_{p-s} \boldsymbol{u}_{p-s}^{-}, \quad s=1 \ldots p-1, \\
& \boldsymbol{\chi}^{+}(p)=p \sqrt{2 p} \boldsymbol{f}_{p}, \quad \chi^{-}(p)=(-1)^{p+1} p \sqrt{2 p} \boldsymbol{f}_{0} .
\end{aligned}
$$

This identification establishes an isomorphism between $\mathcal{T}_{\mathfrak{q}}$ and the center of $\overline{\mathcal{U}}_{\mathfrak{q}} s \ell(2)$ as associative commutative algebras.

Under the identification (5.1), $\mathcal{T}_{\mathfrak{q}}$ coincides with the center of $\overline{\mathcal{U}}_{\mathfrak{q}} s \ell(2)$ as the representation of $S L(2, \mathbb{Z})$. In particular, the relations $\mathcal{S}\left(\chi^{ \pm}(s)\right)=\widehat{\boldsymbol{\phi}}^{ \pm}(s)$ for $s=0 \ldots p$ in the center are parallel to the relations (4.10) in $\mathcal{T}_{\mathfrak{q}}$. The Gaussian element $\boldsymbol{v}$ in notations of [6]

$$
\boldsymbol{v}=\sum_{s=0}^{p}(-1)^{s+1} \mathfrak{q}^{-\frac{1}{2}\left(s^{2}-1\right)} \boldsymbol{e}_{s}+\sum_{s=1}^{p-1}(-1)^{p} \mathfrak{q}^{-\frac{1}{2}\left(s^{2}-1\right)} \frac{\mathfrak{q}^{s}-\mathfrak{q}^{-s}}{\sqrt{2 p}} \widehat{\boldsymbol{\varphi}}(s),
$$

where $\widehat{\varphi}(s)=\frac{p-s}{p} \widehat{\boldsymbol{\phi}}^{+}(s)-\frac{s}{p} \widehat{\boldsymbol{\phi}}^{-}(p-s)$ for $1 \leqslant s \leqslant p-1$ coincides with the ribbon element of $\bar{u}_{\mathfrak{q}} s \ell(2)$.

\section{DISCUSSION}

We identified the representation of DAHA that gives the Verlinde algebra of $(1, p)$ logarithmic conformal field models. The center of $\overline{\mathcal{U}}_{\mathfrak{q}} s \ell(2)$ coincides with the symmetrization of $\mathcal{Z}$ and $C=-\left(X+X^{-1}\right)$ coincides with the $\overline{\mathcal{U}}_{\mathfrak{q}} s \ell(2)$ Casimir element. Probably the 
whole representation $Z$ can be realized in $\overline{\mathcal{U}}_{\mathfrak{q}} s \ell(2)$ such that $X$ would be realized by a multiplication with a $\overline{\mathcal{U}}_{\mathfrak{q}} s \ell(2)$ element.

Another interesting direction of investigations is to find a realization of $\mathcal{H}$ on $(1, p)$ logarithmic conformal field model conformal blocks. This can also be useful in boundary conformal field theories. The Ishibashi and Cardy boundary states can probably be identified with eigenvectors of operators $C=-\left(X+X^{-1}\right)$ and $H=-\left(Y+Y^{-1}\right)$ respectively.

Acknowledgments. We are grateful to T. Suzuki, M. Kasatani and T. Kuwabara for many valuble discussions and A.M. Semikhatov for the useful discussions and comments on a presentation of our results. The work of GM was supported by the RFBR Grant 07-0100523. The work of IYuT was supported in part by LSS-4401.2006.2 grant, the RFBR Grant 05-02-17217 and the "Dynasty" foundation.

\section{APPENDiX A. PROOF OF $\mathcal{S}^{2}=\mathfrak{q} T^{-1}$}

We calculate coefficient in front of $e_{j}$ in A.1 and coefficient in front of $w_{j}$ in A.2.

A.1. The coefficient in front of $e_{j}$. The substitution of (2.26), (2.28), (2.30) in (3.5) gives the coefficient in front of $e_{j}$

$$
\begin{aligned}
\mathrm{f}_{1, s}^{(w)} \mathrm{u}_{j, 1}^{(e)}+\mathrm{f}_{p, s}^{(w)} \mathrm{u}_{j, p}^{(e)}+\mathrm{f}_{p+1, s}^{(w)} \mathrm{u}_{j, p+1}^{(e)}+\mathrm{f}_{2 p, s}^{(w)} \mathrm{u}_{j, 2 p}^{(e)}+\mathrm{f}_{p, s}^{(e)} \mathrm{f}_{j, p}^{(e)}+\mathrm{f}_{2 p, s}^{(e)} \mathrm{f}_{j, 2 p}^{(e)}+ \\
+\left(\sum_{r=2}^{p-1}+\sum_{r=p+2}^{2 p-1}\right)(\underbrace{\mathrm{f}_{r, s}^{(w)} \mathrm{u}_{j, r}^{(e)}+\mathrm{f}_{r, s}^{(m)} \mathrm{k}_{j, r}^{(e)}}_{A}),
\end{aligned}
$$

where all numbers $u, k, f$ are given in (2.27), (2.29), (2.31). A simplification of the underbraced expression gives

$$
\begin{aligned}
& \text { for } j \neq 1, p, p+1,2 p \text { : } \\
& A=\frac{(-1)^{s+j} \mathfrak{q}^{2}}{2 p}\left(\mathfrak{q}^{r-1}[s, r-1][r, j]-[s, r-1][r, j-1]\right)+ \\
& +\frac{(-1)^{s+j} \mathfrak{q}^{s}}{2 p}\left(\mathfrak{q}[s, r][r, j-1]-\mathfrak{q}^{r}[s, r][r, j]\right), \\
& \text { for } j=1 \text { : } \\
& A=\frac{(-1)^{s+1} \mathfrak{q}^{2}}{2 p}\left(\mathfrak{q}^{r-1}[s, r-1][r, 1]-[s, r-1]\right)+\frac{(-1)^{s+1} \mathfrak{q}^{s}}{2 p}\left(-\mathfrak{q}\{s, r\}-\mathfrak{q}^{r}[s, r][r, 1]\right), \\
& \text { for } j=p+1 \text { : } \\
& A=\frac{(-1)^{s+p+1} \mathfrak{q}^{2}}{2 p}\left(\mathfrak{q}^{r-1}[s, r-1][r, p+1]-[s+p, r-1]\right)+
\end{aligned}
$$




$$
\begin{aligned}
+ & \frac{(-1)^{s+p+1} \mathfrak{q}^{s}}{2 p}\left(\mathfrak{q}\{s+p, r\}-\mathfrak{q}^{r}[s, r][r, p+1]\right), \\
\text { for } j=p: & \\
A=\frac{(-1)^{s+p} \mathfrak{q}^{2}}{2 p}\left(\mathfrak{q}^{r-1}[s+p, r-1]-\right. & {[s, r-1][r, p-1])+ } \\
& +\frac{(-1)^{s+p} \mathfrak{q}^{s}}{2 p}\left(\mathfrak{q}[s, r][r, p-1]-\mathfrak{q}^{r}\{s+p, r\}\right),
\end{aligned}
$$

for $j=2 p$ :

$$
A=\frac{(-1)^{s} \mathfrak{q}^{2}}{2 p}\left(\mathfrak{q}^{r-1}[s, r-1]-[s, r-1][r, 2 p-1]\right)+\frac{(-1)^{s} \mathfrak{q}^{s}}{2 p}\left(\mathfrak{q}[s, r][r, 2 p-1]+\mathfrak{q}^{r}\{s, r\}\right) .
$$

Then the simplification of (A.1) gives coefficients in (3.5) in front of $e_{s}$. Explicitly, the summation in $r$ of different terms in $A$ is given by

$$
\begin{aligned}
& \left(\sum_{r=2}^{p-1}+\sum_{r=p+2}^{2 p-1}\right) q^{r-1}[s, r-1][r, j]=\left(p-\frac{1}{4}((s+j+1 \bmod 2 p)+(s-j+1 \bmod 2 p)+\right. \\
& +(s+j-1 \bmod 2 p)+(s-j-1 \bmod 2 p)))\left(1+(-1)^{s+j}\right)+p \frac{\{j\}}{[j]}\left(\delta_{s+j, 2 p}-\delta_{s-j, 0}\right), \\
& \left(\sum_{r=2}^{p-1}+\sum_{r=p+2}^{2 p-1}\right)[s, r-1][r, j]=\left(1+(-1)^{s+j+1}\right)\left(p-\frac{1}{2}((s+j \bmod 2 p)+\right. \\
& +(s-j \bmod 2 p))) \\
& \left(\sum_{r=2}^{p-1}+\sum_{r=p+2}^{2 p-1}\right)[s, r][r, j]=[s]\left((-1)^{s+j}-1\right), \quad j \neq p, 2 p \\
& \left(\sum_{r=2}^{p-1}+\sum_{r=p+2}^{2 p-1}\right) \mathfrak{q}^{r}[s, r][r, j]=\frac{2 p\left(\delta_{s, 2 p-j}-\delta_{s, j}\right)}{\mathfrak{q}^{j}-\mathfrak{q}^{-j}}-\mathfrak{q}[s]\left(1+(-1)^{j+s}\right), \quad j \neq p, 2 p, \\
& \left(\sum_{r=1}^{p-1}+\sum_{r=p+1}^{2 p-1}\right)[s, r]=\left(1-(-1)^{s}\right)(p-(s \bmod 2 p)) \\
& \sum_{r=1}^{2 p}\{s, r\}=0 \\
& \left(\sum_{r=1}^{p-1}+\sum_{r=p+1}^{2 p-1}\right) \mathfrak{q}^{r}[s, r]=\left(1+(-1)^{s}\right)\left(p-\frac{((s+1) \bmod 2 p)+((s-1) \bmod 2 p)}{2}\right) \\
& \sum_{r=1}^{2 p} \mathfrak{q}^{r}\{s, r\}=\left(1+(-1)^{s}\right)\left(\frac{((s-1) \bmod 2 p)-((s+1) \bmod 2 p)}{2}\right) .
\end{aligned}
$$


A.2. The coefficient in front of $w_{j}$. The substitution of (2.26), (2.28), (2.30) in (3.5) gives the coefficient in front of $w_{j}$

$$
\begin{aligned}
\mathrm{f}_{1, s}^{(w)} \mathrm{u}_{j, 1}^{(w)}+\mathrm{f}_{p, s}^{(w)} \mathrm{u}_{j, p}^{(w)}+\mathrm{f}_{p+1, s}^{(w)} \mathrm{u}_{j, p+1}^{(w)}+\mathrm{f}_{2 p, s}^{(w)} \mathrm{u}_{j, 2 p}^{(w)}+\mathrm{f}_{p, s}^{(e)} \mathrm{f}_{j, p}^{(w)}+\mathrm{f}_{2 p, s}^{(e)} \mathrm{f}_{j, 2 p}^{(w)}+ & \\
& +\left(\sum_{r=2}^{p-1}+\sum_{r=p+2}^{2 p-1}\right)(\underbrace{\mathrm{f}_{r, s}^{(w)} \mathrm{u}_{j, r}^{(w)}+\mathrm{f}_{r, s}^{(m)} \mathrm{k}_{j, r}^{(w)}}_{A}),
\end{aligned}
$$

where all numbers $u, k, f$ are given in (2.27), (2.29), (2.31). A simplification of the underbraced expression gives

$$
\begin{gathered}
A=\frac{\mathfrak{q}^{2}(-1)^{s+j}\{1, j-1\}}{p\left(\mathfrak{q}^{j-1}+\mathfrak{q}^{-j+1}\right)}[s, r-1][r-1, j-1]-\frac{(-1)^{s+j}\{1, j\} \mathfrak{q}^{2}}{p\left(\mathfrak{q}^{j}+\mathfrak{q}^{-j}\right)} \mathfrak{q}^{r-1}[s, r-1][r-1, j]+ \\
+\frac{\mathfrak{q}^{s}(-1)^{s+j}}{2 p}\left(\mathfrak{q}^{r}\{s, r\}\{r, j\}-\mathfrak{q}\{s, r\}\{r, j-1\}+\mathfrak{q}^{r}[s, r][r, j]\{1, j\}-\mathfrak{q}[s, r][r, j-1]\{1, j-1\}\right)
\end{gathered}
$$

Then the simplification of (A.2) gives coefficients in (3.5) in front of $w_{s}$. Explicitly, the summation in $r$ of different terms in A.3 is given by

$$
\begin{gathered}
\left(\sum_{r=2}^{p-1}+\sum_{r=p+2}^{2 p-1}\right)[s, r-1][r-1, j]=[s]\left(1-(-1)^{s+j}\right), \quad j \neq p, 2 p, \\
\left(\sum_{r=2}^{p-1}+\sum_{r=p+2}^{2 p-1}\right) \mathfrak{q}^{r-1}[s, r-1][r-1, j]=\frac{2 p\left(\delta_{s, 2 p-j}-\delta_{s, j}\right)}{\mathfrak{q}^{j}-\mathfrak{q}^{-j}+}+\mathfrak{q}^{-1}[s]\left(1+(-1)^{j+s}\right), \quad j \neq p, 2 p, \\
\left(\sum_{r=2}^{p-1}+\sum_{r=p+2}^{2 p-1}\right) \mathfrak{q}^{r}\{s, r\}\{r, j\}=\left(\frac{p\left(\delta_{s+j, 2 p}+\delta_{s-j, 0}\right)-2}{\mathfrak{q}^{j}+\mathfrak{q}^{-j}}-\mathfrak{q}\{s\}\right)\{1, j\}\left(1+(-1)^{j+s}\right), \\
\left(\sum_{r=2}^{p-1}+\sum_{r=p+2}^{2 p-1} \sum_{r=2}^{p-1}+\sum_{r=p+2}^{2 p-1}\right)\{s, r\}\{r, j\}=\{s, 1\}\{1, j\}\left((-1)^{s+j}-1\right), \\
\left(\sum_{r=2}^{p-1}+\sum_{r=p+2}^{2 p-1}\right)[s, r][r, j]=[s]\left((-1)^{s+j}-1\right), \quad j \neq p, 2 p .
\end{gathered}
$$

\section{REFERENCES}

[1] A.M. Semikhatov, Factorizable ribbon quantum groups in logarithmic conformal field theories, [hepth/0705.4267].

[2] V. Gurarie, Logarithmic operators in conformal field theory, Nucl. Phys. B410 (1993) 535 [hep-th/ 9303160]. 
[3] M.R. Gaberdiel and H.G. Kausch, A rational logarithmic conformal field theory, Phys. Lett. B386 (1996) 131-137 [hep-th/9606050].

[4] B.L. Feigin, A.M. Gainutdinov, A.M. Semikhatov, and I.Yu. Tipunin, Kazhdan-Lusztig correspondence for the representation category of the triplet W-algebra in logarithmic CFT, math.QA/0512621.

[5] D. Kazhdan and G. Lusztig, Tensor structures arising from affine Lie algebras, I, J. Amer. Math. Soc. 6 (1993) 905-947; II, J. Amer. Math. Soc. 6 (1993) 949-1011; III, J. Amer. Math. Soc. 7 (1994) 335-381; IV, J. Amer. Math. Soc. 7 (1994) 383-453.

[6] B.L. Feigin, A.M. Gainutdinov, A.M. Semikhatov, and I.Yu. Tipunin, Modular group representations and fusion in logarithmic conformal field theories and in the quantum group center, Commun. Math. Phys. 265 (2006) 47-93 [hep-th/0504093].

[7] M.R. Gaberdiel and H.G. Kausch, Indecomposable fusion products, Nucl. Phys. B477 (1996) 293-318 [hep-th/9604026].

[8] J. Fuchs, S. Hwang, A.M. Semikhatov, and I.Yu. Tipunin, Nonsemisimple fusion algebras and the Verlinde formula, Commun. Math. Phys. 247 (2004) 713-742 [hep-th/0306274].

[9] M. Flohr and H. Knuth, On Verlinde-Like Formulas in $c_{p, 1}$ Logarithmic Conformal Field Theories, math-ph/0705.0545.

[10] M. Flohr, Bits and pieces in logarithmic conformal field theory, Int. J. Mod. Phys. A18 (2003) 44974592 [hep-th/0111228].

[11] I. Cherednik, Double Affine Hecke Algebras, 2004.

[12] I. Cherednik, Private communication, Kyoto, 2004.

GM: Moscow Institute of Physics and Technology

GEORG21A@YAHOO.COM

IYT: LeBedev Physics Institute

T I PUNIN@LPI . RU

$p p^{\prime}$ models and quantum groups - August 30, $2021-1: 21$ 